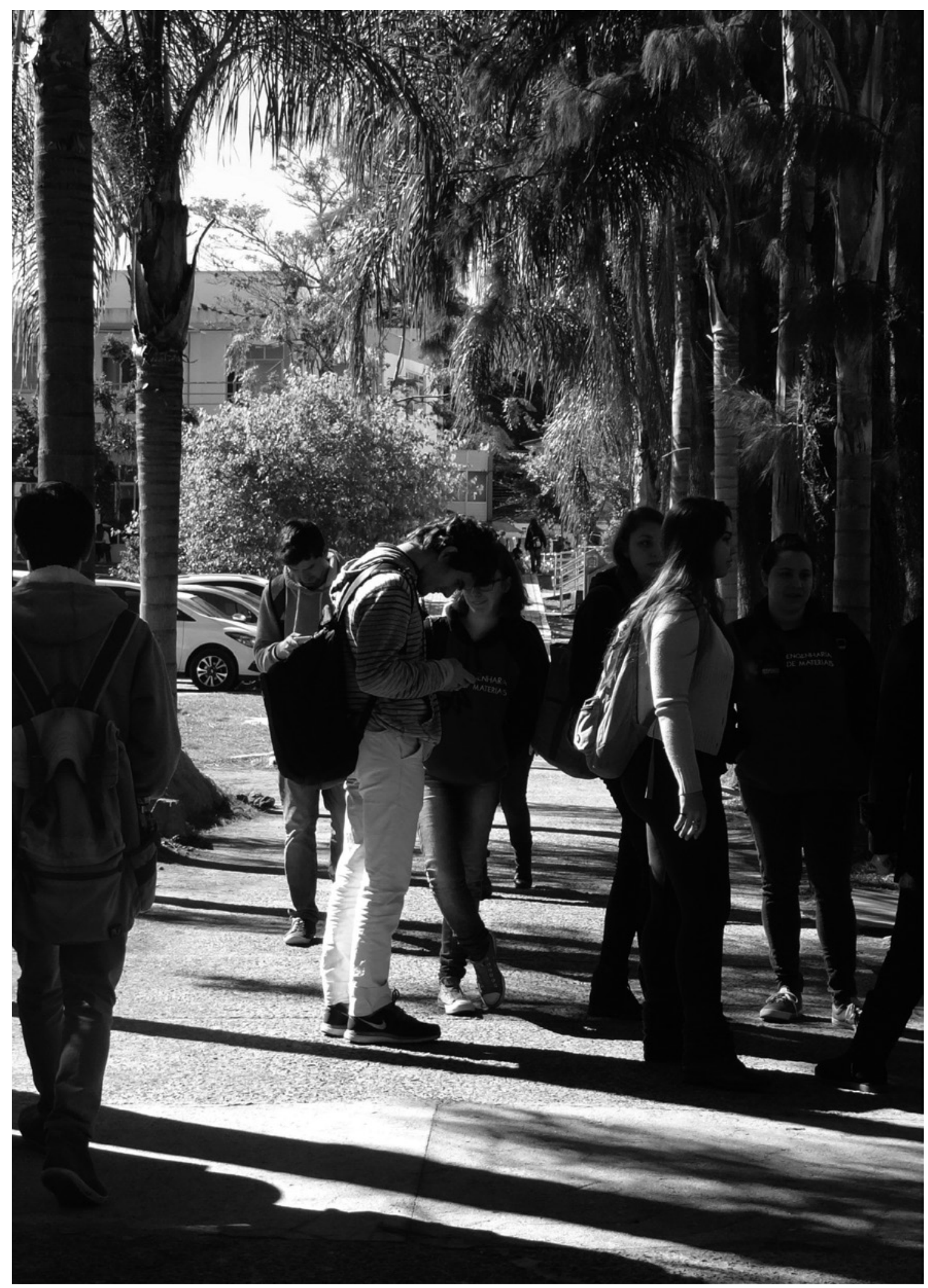

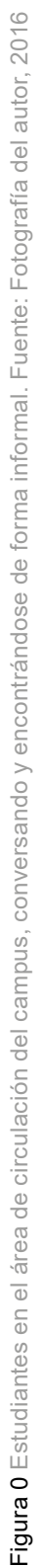



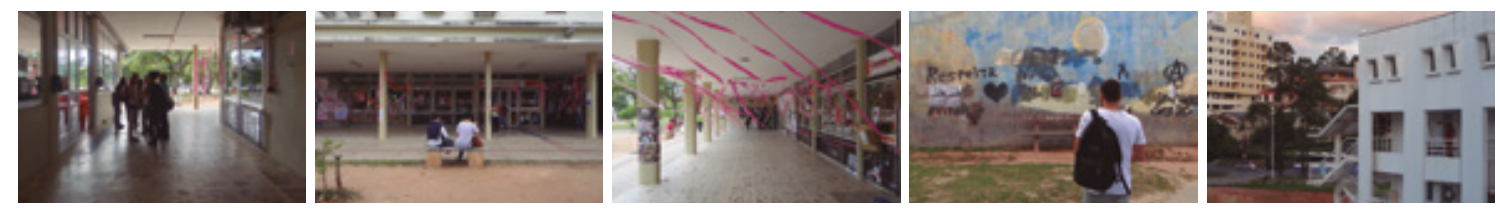

Secuencia: Ejercicio de levantamiento de la UFSC

Fotos: Gustavo Peters

\title{
COPRESENCIA, INTERACCIÓN Y DIVERSIDAD: ANÁLISIS SOCIOESPACIAL EN EL CAMPUS UNIVERSITARIO'
}

\author{
COPRESENCE, INTERACTION AND DIVERSITY: SOCIO-SPATIAL ANALYSIS ON A \\ UNIVERSITY CAMPUS ${ }^{1}$
}

\section{Gustavo Peters de Souza², Renato Tibiriçá de Saboya ${ }^{3}$}

RESUMEN

Este artículo analiza las características de los espacios libres que incentivan la copresencia, interacción y diversidad de perfiles de usuarios en ocho lugares seleccionados en el campus principal de la Universidad Universidad Federal de Santa Catarina, Brasil. La investigación adopta métodos cuantitativos y cualitativos, explorando el potencial de la configuración y organización espacial para crear áreas educacionales que produzcan encuentros informales. Los datos fueron recolectados por medio de la observación sistemática, que generó mapas de comportamiento, y de la aplicación de cuestionarios que recogieron información sobre la diversidad y las razones para usar cada lugar. El estudio muestra que las áreas con mayor intensidad de copresencia e interacción son los espacios cercanos a los servicios académicos, bien configurados espacialmente y que presentan altos valores en las medidas sintácticas locales, aquí aplicadas: "integración" y "elección". En cuanto a la variable "diversidad", la "elección global" mostró ser capaz de captar dicha diversidad en espacios situados entre centros académicos, mientras que la diversidad entre cursos fue mejor captada por la "elección local", "integración global" e "integración local", en ese orden.

Palabras clave: campus universitario, interacción social, educación informal, espacio abierto, comportamiento social.

\section{ABSTRACT}

This article analyzes the characteristics of free spaces that encourage copresence, interaction and diversity of user profiles, in eight selected locations at the main campus of the Federal University of Santa Catarina, Brazil. The research adopted quantitative and qualitative methods to explore the potential of configuration and spatial organization in creating educational areas that produce informal encounters. Data were collected through systematic observation, which generated behavioral maps, and through the application of questionnaires, which gathered information about diversity and the reasons for using each location. The study shows that the areas with the highest intensity of copresence and interaction are the spaces close to the academic services. They are well-configured spatially and have high values in both the local syntactic measures of "integration" and "choice". Regarding the variable "diversity", the measure of "global choice" was able to capture the diversity in spaces located between academic centers, while the diversity among courses was best obtained through the measures of "local choice", "global integration" and "local integration", in that order.

Keywords: university campus, social interaction, informal education, open space, social behavior.

Artículo recibido el 10 de agosto de 2017 y aceptado el 18 de diciembre de 2017 DOI: https://doi.org/10.22320/07196466.2017.35.052.06

[1] Este artículo está basado en los resultados de investigación de la tésis de magister del autor titulada: "COPRESENCIA, INTERACCIÓN Y DIVERSIDAD: ANÁLISIS SOCIOESPACIAL EN EL CAMPUS DE LA UNIVERSIDAD FEDERAL DE SANTA CATARINA" financiada por BECA CAPES -DS 2015-2017, Universidad Federal Santa Catarina, Florianápolis, Brasil, 19 de junio de 2017.

[2] Universidad Federal de Santa Catarina - UFSC, Florianápolis, Brasil. arq.gustavopeters@gmail.com

[3] Universidad Federal de Santa Catarina -UFSC, Florianápolis, Brasil.rtsaboya@gmail.com 
La transmisión y producción del conocimiento en las universidades ocurre tanto formal, por medio de clases, seminarios y otras actividades institucionales, como informalmente, por medio de intercambios no planificados fuera de las aulas de clase, en las áreas externas del campus. El concepto de "campus educacional" propone considerar este proceso de aprendizaje como fruto de la comunicación que incluye, pero transciende los espacios formales de enseñanza. Calvo-Sotelo (2010; 2014) y Halsband (2005), por ejemplo, consideran la importancia de la vitalidad de las áreas universitarias para la interacción y de su función en la tarea de promover el contacto entre las personas por medio de espacios compartidos colectivamente. De forma similar, otros autores, como Sosa (2011) y Hertzberger (2009), proponen directrices para transformar los espacios externos en áreas creativas que estimulen actitudes innovadoras a través de encuentros espontáneos.

Para que ocurran estos encuentros aleatorios, una condición básica es la "copresencia", concepto que aparece en los estudios de Goffman (1963:17) y se define como una condición en la cual los individuos sienten que están lo suficientemente próximos para ser percibidos $y$, por lo tanto, comparten el mismo espacio, al mismo tiempo. Las interacciones sociales en el espacio necesitan de individuos copresentes, quiere decir, que estén lo suficientemente próximos para percibirse e intercambiar información. A pesar de que la copresencia no sea condición suficiente para la interacción, es una condición necesaria.

Tanto la copresencia como la interacción pueden ocurrir entre personas de la misma clase socioeconómica o, de manera general, de un mismo grupo social. En estos casos de gran homogeneidad entre los sujetos, el potencial de intercambio de información atípica y no convencional, el conocimiento del otro y el incentivo a la tolerancia son desperdiciados. En el caso de los espacios universitarios se piensa, además de eso, que esta mayor diversidad está vinculada al intercambio de ideas entre disciplinas, mayor generación de nuevas ideas y la combinación entre conocimientos complementarios $y$, consecuentemente, mayor capacidad de innovación (Penn, Desyllas y Vaughan, 1999; Sosa, 2011; Allen y Henn, 2013; Wineman et al., 2014)

Entre tanto, a pesar de ser considerada positiva por diversos autores (Jacobs, 2009; Alexander et al., 1977; Gehl, 2011; Talen, 2006, entre otros) y de ser una preocupación principal en los estudios urbanos desde al menos la década de los 60, Talen (2006) considera que la diversidad aún es una variable poco estudiada: "A pesar de haberse escrito mucho sobre la diversidad, el vínculo entre la planificación de las ciudades -definida aquí en su sentido tradicional, como un quehacer preocupado del diseño de las ciudades-y la diversidad de los lugares ha sido poco estudiado"4 (Talen, 2006: 234). La literatura próxima a este tema muestra que este se toma más en consideración en los estudios de transporte (Cervero y Duncan, 2006; Ewing y Cervero, 2010) y de salud (Boar- 
net et al., 2011; Van Dyck et al., 2012), en los cuales se prueba la relación entre diversidad de usos del suelo y la adopción de la caminata como medio de transporte o de placer. Asimismo, pocos son los estudios que problematizan y reflejan las maneras de medir diversidad y la mayoría de ellos importa acríticamente medidas derivadas de la Ecología, salvo algunas pocas excepciones, como Smith y Wilson, 1996; Song, Merlin y Rodriguez, 2013; y Gehrke y Clifton, 2014.

Mientras, estos estudios se concentran en la diversidad de usos del suelo. Talen (2008) es una de las pocas en abocarse a la diversidad de perfiles de usuario, aunque adopta el índice de Simpson que, como se verá más adelante, genera problemas operacionales significativos. Adicionalmente, sus estudios se concentran en el espacio urbano en general, utilizando el sector censitario como unidad espacial. En el caso concreto de los espacios universitarios, esta carencia de estudios sobre la diversidad de usuarios y su relación con el espacio queda evidenciada, especialmente considerando que estos lugares poseen características morfológicas y dinámicas de uso bien distintos a las áreas urbanas en general, especialmente en Brasil, donde son comunes los grandes campus universitarios separados de la red urbana. Esta situación perjudica la transposición de conclusiones relativas a la ciudad como un todo para los campus.

Incluso en lo que respecta al estudio de los espacios abiertos de forma general, las áreas universitarias aparecen solo ocasionalmente por medio de análisis de la relación del campus con la ciudad, como en los trabajos de Lee, Han y Kim (2014) y de Halsband (2005). Sólo algunas investigaciones más recientes, realizadas en universidades u organizaciones empresariales, consideran las consecuencias de la configuración espacial como un factor influyente del proceso de reproducción del conocimiento y de la innovación -entre las que destacan Abu-Ghazzeh (1999), Peponis et al. (2007) y Wineman et al. (2014), a pesar de que no se enfocan en el estudio de la diversidad de usuarios.

El campus de la Universidad Federal de Santa Catarina, Brasil, objeto del estudio que aquí se expone, elaboró su primer plan directivo entre 1956 y 1957, de la mano del arquitecto Hélio de Queiróz Duarte y el ingeniero Ernesto R. de Carvalho. Sin embargo, sólo en 1970 surgió el primer proyecto de un espacio con características que podrían promover la interacción entre los alumnos. La Plaza de la Ciudadanía fue diseñada por el equipo del paisajista Roberto Burle Marx, y fue el principal elemento organizador de todos los sectores, flujos y edificios existentes. A partir de 1994 y 1998, se creó un plan de organización de los espacios del campus. Este Plan Directivo Participativo del Campus preveía la absorción de espacios libres para atender la sucesiva demanda de aumento de las áreas y también estaba comprometido a diseñar áreas de encuentro, de disfrute y áreas verdes; sin embargo, fue objeto de revisiones y estudios en 2005 , 2009, 2010 y 2011. La configuración actual del campus, resultado de estas diversas propuestas, corresponde a una universidad inserta en la red urbana, conformada por bloques aislados por departamentos, los cuales se interconectan mayormente a través de vías vehiculares y caracterizada por una fragmentación de iniciativas diferentes, que no siempre se integraron adecuadamente.
En concreto, este trabajo examina los conceptos de copresencia, interacción y diversidad, y busca reconocer las características espaciales que incentivan su desarrollo en el Campus central de la Universidad Universidad Federal de Santa Catarina, Brasil. Para ello, se analizan ocho lugares alrededor de centros de gravitación (cafeterías, terminales bancarios, servicios de copias y áreas comunes), cruzando sus características espaciales con los niveles de los fenómenos de interés observados in situ. Se considera que los resultados pueden contribuir a la comprensión de los posibles estándares de comportamiento de los usuarios y en la futura planificación de los espacios abiertos en los campus universitarios, en aras de promover una mayor intensidad de la comunicación y compartir el conocimiento.

\section{COPRESENCIA, INTERACIÓN, DIVERSIDAD Y ESPACIO}

La "diversidad" es un concepto ya consagrado y central para la teoría urbanística, al menos desde la década de los 60, con el trabajo de Jacobs (2009, publicado originalmente en 1961). En él, la autora defiende la necesidad de la diversidad de usos, de las personas que utilizan el espacio en diferentes horarios, y de las edades de las edificaciones, que generarían condiciones diferentes de acceso al espacio para empresas y los habitantes con también distintos poderes adquisitivos.

Talen (2006) compila una serie de razones por las cuales la diversidad sería importante: amplía la vitalidad urbana y la capacidad de innovación de las empresas, incentiva la creatividad, sustenta un mayor crecimiento económico, garantiza el acceso más justo a los recursos ofrecidos por la ciudad, y mejora los procesos de renovación cultural y ecológica, entre otros supuestos efectos. Aun cuando estas afirmaciones no sean consensualmente aceptadas, como la misma autora deja claro, parece haber pocas dudas de que una mayor diversidad de grupos sociales coexistiendo e interactuando en el espacio puede proporcionar existencias más nutridas, incentivar la tolerancia, el conocimiento sobre el otro y sus diferentes realidades, y el contacto con otros valores $y$ formas de ver el mundo.

A partir de esa noción, se desarrollan otras, como la "copresencia" en tiempo y espacio, y la "interacción". La proximidad, condición fundamental para ambas categorías y factor relevante en las relaciones sociales, aparece en los estudios de Hall (1966), que clasifica la distancia entre las personas durante la interacción, definiendo, así, el nivel de relación que tienen entre sí. Posteriormente, las investigaciones empíricas de Allen (1997), analizando la estructura organizacional de las organizaciones, definen que la información sólo logra ser compartida de manera eficaz a un máximo de $50 \mathrm{~m}$ de distancia, más allá de lo cual la intensidad de la interacción disminuye drásticamente.

La copresencia ha sido extensamente estudiada por la Sintaxis Espacial, teoría creada a finales de la década de los 70 e inicios de la década de los 80, en Inglaterra (HiIlier et al., 1987; Hillier y Hanson, 1984). Estudios de con- 
figuración del espacio han demostrado empíricamente que la cantidad de personas en cada lugar, caminando o paradas, está directamente vinculada al papel que aquel espacio desempeña como un todo (Hillier et al., 1993; Penn et al., 1998; Turner et al., 2001; Hillier y lida, 2005). A pesar de su (aparente) simplicidad, esta noción había sido poco explorada hasta dichas décadas, por falta de medios rigurosos para describir su papel. Las medidas de integración y elección, aliadas al concepto de línea axial y, más tarde, de análisis angular por segmentos, superaron este problema. Investigaciones como las de Greene y Penn (1997), Abu-Ghazzeh (1999) y Adhya (2009) ya incluyen los análisis sintácticos en sus investigaciones en áreas universitarias. Por otro lado, la diversidad de usuarios permanece poco explorada en la Sintaxis Espacial.

El papel de la diversidad en la comunicación informal y espontánea, así como sus efectos, puede comprenderse mejor desde el concepto de vínculos fuertes y frágiles de Granovetter, según el cual la fuerza de un vínculo interpersonal es "una combinación de la cantidad de tiempo, intensidad emocional, intimidad (confianza mutua) y servicios mutuos que caracterizan el vínculo" ${ }^{5}$ (1973:1361). Los lazos fuertes tienden a implicar comunicación entre personas de la misma red social, ya que los individuos con este tipo de relación probablemente tienen muchos contactos y/o amigos en común. Por otro lado, los vínculos frágiles e indirectos se asocian más frecuentemente a procesos comunicativos que transponen barreras de grupos específicos y, por ello, tienen mayor capacidad de diseminación de la información. De esta forma, este tipo es especialmente capaz de crear puentes entre disciplinas, organizaciones, grupos sociales y comunidades en general, contribuyendo con los procesos de integración del conocimiento que traen innovación. Otros autores también clasifican esta comunicación en sus investigaciones relacionadas al componente espacial, como "comunicación/coordinación" o "inspiración" (Allen y Henn, 2013); planificada o por serendipia (Peponis et al., 2007); directa o indirecta (Wineman et al., 2014); y por medio de modelos largos o cortos (Hillier y Penn, 1991).

Los vínculos frágiles, necesarios para la transmisión del conocimiento innovador, ocurren con más intensidad con la presencia de personas de diferentes áreas interdisciplinarias, ya que las expone a ideas nuevas o desconocidas, diferentes de aquellas directamente más accesibles que las que acostumbran circular en sus núcleos sociales donde los vínculos fuertes son más numerosos. Sin embargo, la representación del espacio en estos estudios no tiene el mismo refinamiento teórico y operacional que aquel alcanzado por los estudios arquitectónicos y morfológicos.

En este trabajo, por tanto, se une la interacción y diversidad de usuarios con una descripción más refinada del espacio, especialmente aquella desarrollada en el ámbito de la Sintaxis Espacial. De este modo, se espera contribuir a la temática, a través de la unión de dos campos de estudio diferentes, con el fin de revelar nuevas relaciones entre estos fenómenos, específicamente en lo que se refiere a los ambientes universitarios.

\section{MÉTODO}

El primer paso del estudio fue seleccionar los lugares del campus a ser analizados. Para cada uno de estos lugares, se realizó el levantamiento in situ de variables que representan los fenómenos de interés (copresencia, interacción y diversidad), así como el espacio y sus relaciones (integración, elección y visibilidad). Estas variables fueron, entonces, mapeadas y agregadas para el análisis. A continuación, se elaboró un cuestionario para identificar el perfil de los usuarios de los ocho lugares, con vistas a determinar la diversidad de esos usuarios, tanto por centro de enseñanza como por cursos.

La labor abarcó análisis visuales, permitidos por los mapas elaborados, y análisis cuantitativos a través de la correlación, basados en la inclusión de las variables en tramos, dentro de cada lugar. Ambos procedimientos buscaron identificar patrones asociativos recurrentes y verificar si las variables espaciales eran capaces de explicar las variables dependientes. Además de eso, en esa etapa fue posible constatar si las correlaciones entre las variables se repetían en todos los lugares o si algunas correlaciones aparecían sólo en algunos de ellos, lo cual dio una idea de la robustez de los resultados en situaciones diferentes.

Finalmente, se crearon medidas de síntesis para cada uno de los ocho lugares, con la intención de verificar si, en el conjunto, era posible encontrar alguna relación entre las variables de interés, o sea, si era posible advertir correlaciones "inter-locales". Los elementos que se presentan a continuación explican estos pasos con más detalle, así como la operación de las variables de interés.

\section{Selección de los lugares de análisis}

Las áreas de análisis fueron seleccionadas basándose en los mapas del campus, que fueron editados y complementados por los autores, y utilizando como criterio de selección los centros de gravitación. Una preocupación metodológica importante fue la de escoger áreas que pudieran ser adecuadamente comparadas entre sí. Por este motivo, una primera decisión fue la de excluir zonas que contuvieran equipos muy grandes y/o importantes, tales como la Biblioteca y el Restaurante Universitario, dado su carácter único y de difícil comparación. Además de eso, estos lugares presentan, forzosamente, diversidad de usuarios, ya que atienden a estudiantes y docentes de todos los cursos de la universidad. Así, se optó por elegir sectores que tuvieran, al menos, un café como punto de interés o gravitacional, en un total de 8 lugares (Figura 1). Estas áreas son naturalmente atractivas para los estudiantes, sin embargo, poseen diferentes intensidades y niveles de diversidad. Algunas están localizadas en el interior de los centros de enseñanza, otras están en los caminos que los conectan y otras se hallan, 
incluso, cercanas a los bordes del campus. Por último, se definió un radio de 50 metros alrededor de cada café, correspondiente a la distancia máxima indicada por Allen (1977) para el movimiento y los encuentros informales, conformándose, de esta manera, áreas de análisis de $100 \mathrm{~m} \times 100 \mathrm{~m}$.

\section{Observación sistemática del comportamiento}

El levantamiento de datos de las variables dependientes en cada uno de los ocho lugares fue realizado por medio de la observación sistemática, mediante filmaciones que generaron información para los mapas de comportamiento. La investigación realizada por Whyte (1980) en la década de los 80, fue pionera, gracias a las filmaciones, en el análisis de la utilización de los espacios públicos. Para Zeisel (1984), observar el comportamiento debe generar datos importantes sobre las actividades de las personas y las acciones necesarias para sustentarlas, posibilitando analizar recurrencias. Por lo demás, el método de observación no atrae la atención $y$, en consecuencia, no ejerce influencia sobre el objeto que será analizado, es aprehendido visualmente de manera eficaz y ofrece datos concretos (Zeisel, 1984). Así, dos personas recorren caminos diferentes con el mismo objetivo, filmando todo durante 3 minutos (Saboya et al., 2014), lapso estipulado por los autores como tiempo útil para recorrer la totalidad del objetivo. Para cada lugar se lleva- ron a cabo tres filmaciones con la finalidad de obtener datos más representativos, una en cada horario, en los intervalos entre clases (mañana: 9:50-10:00; medio día: 11:50-12:00; tarde: 15:50-16:00). Los días se escogieron aleatoriamente durante la semana, en dos semestres regulares y en condiciones climáticas soleadas. De ese modo, cada persona vista en la filmación se introdujo en el mapa, siendo georreferenciada y, posteriormente, clasificada según las caracterizaciones de Zeisel (1984:124):

Actores: alumnos, miembros de la comunidad académica y visitantes.

Actividad: en movimiento, estáticos, sentados, de pie.

Con quién: en grupo, en pareja, solo.

Período de la observación: Mañana, medio día o tarde.

Esta información obtenida por la observación sistemática genera dos tipos de mapa, empleados en los análisis visuales posteriores: mapa de personas estáticas (Figura 3a) y en movimiento, y mapa de densidad de Kernel (Figura 3b), este último usualmente utilizado como alternativa para análisis geográfico del comportamiento de patrones. Además de eso, se agregó los análisis cuantitativos, indicando la cantidad total de personas, proporción entre las personas estáticas y en movimiento, y la proporción de personas en grupos, en parejas o solas.

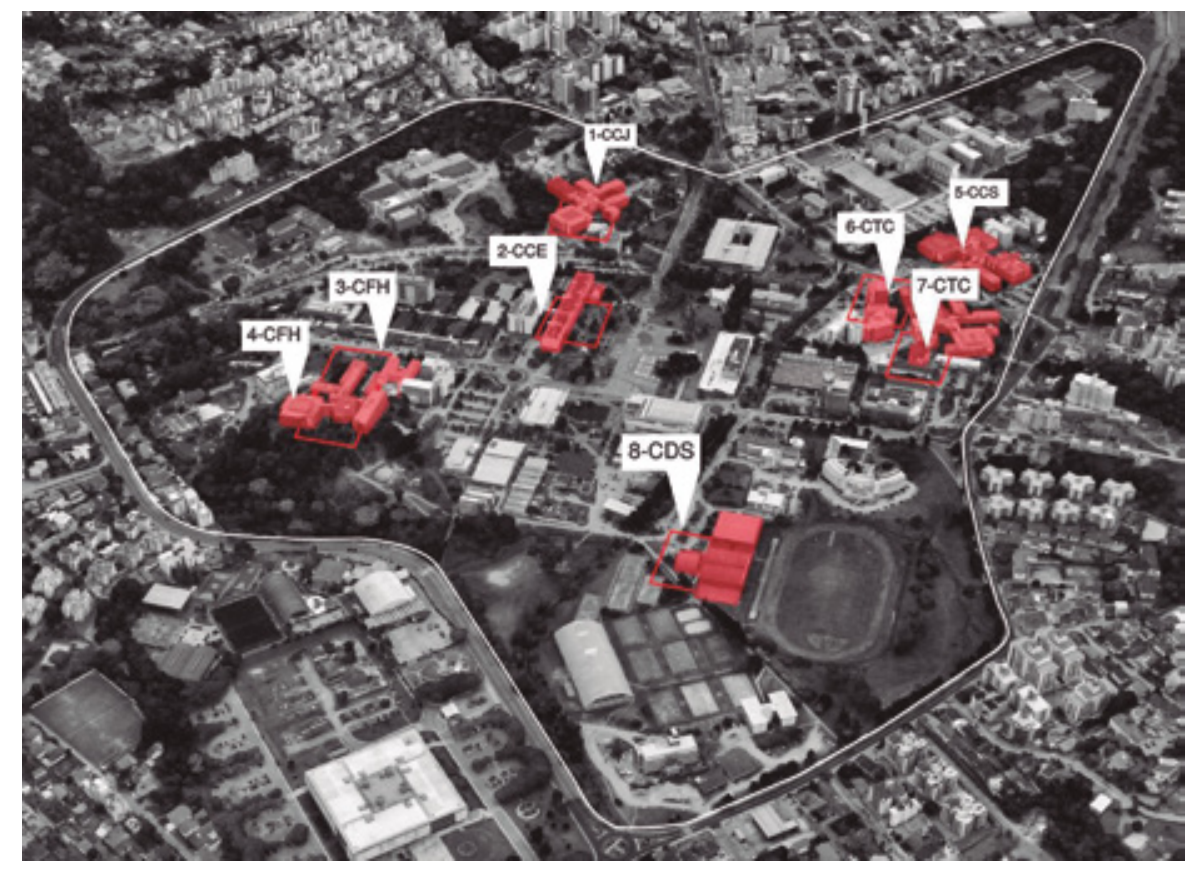

Figura 1 Localización de los objetos de estudio y sus departamentos. Fuente: Elaborado por los autores a partir de imagen de Google Earth y datos do DPAE/UFSC. 2017 

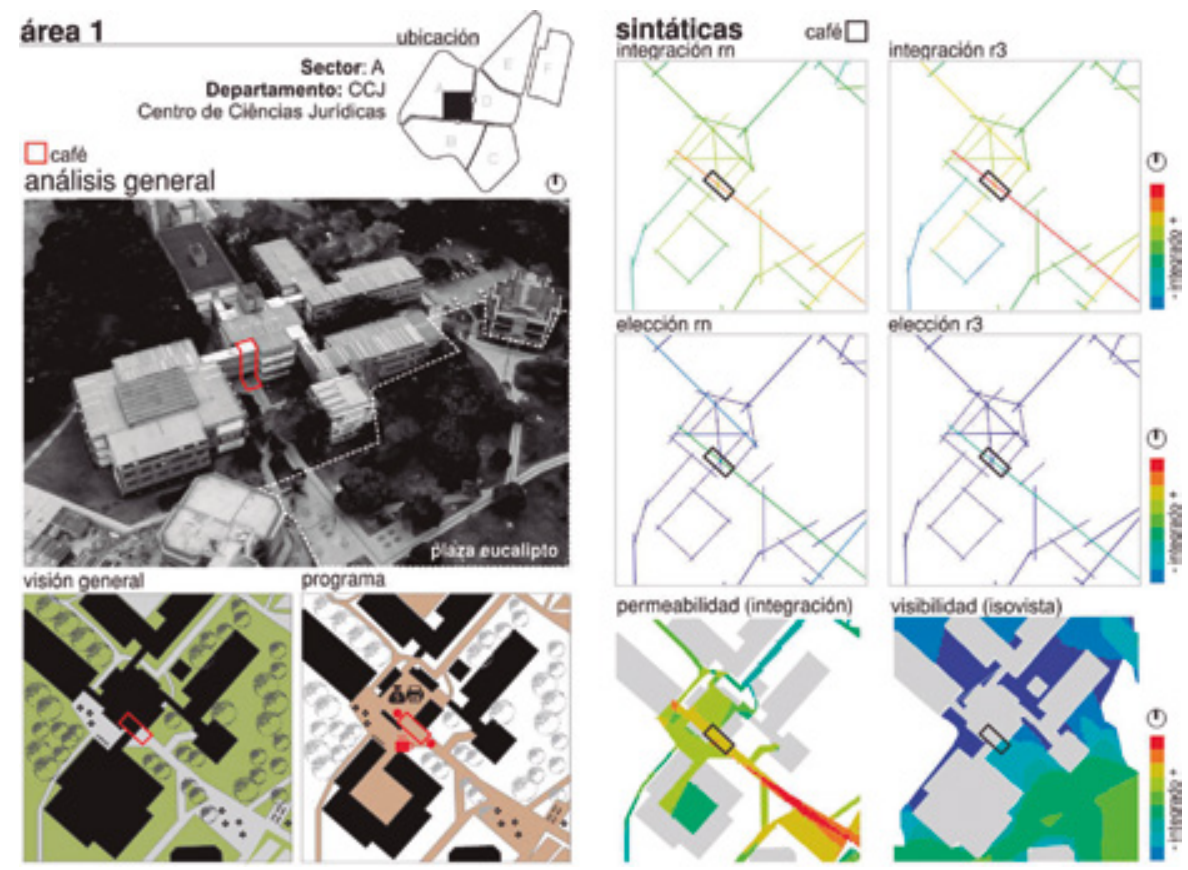

Figura 2 Ficha de contextualización del objetivo 1. Fuente: Elaborado por los autores a partir de datos del DPAE/UFSC y Google Earth

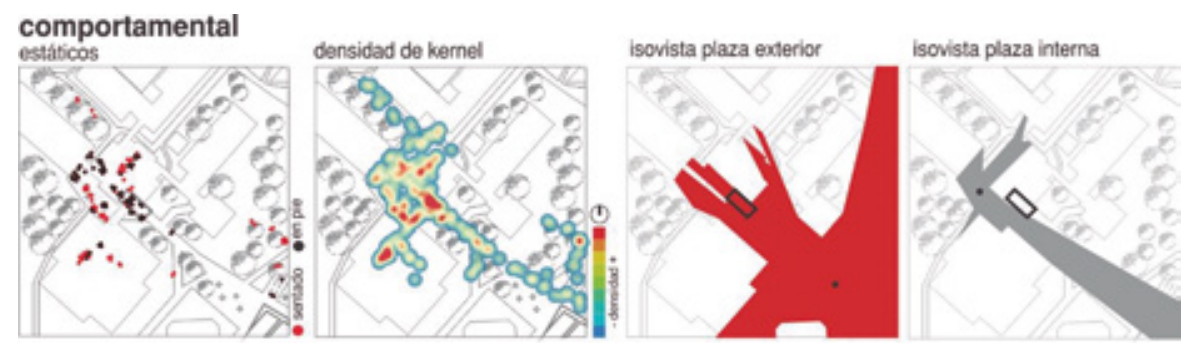

Figura 3 De izquierda a derecha: a) Mapas de comportamiento de personas estáticas; b) Mapa de densidad de Kernel; c) Isovista de la plaza externa; d) Isovista de la plaza interna. Fuente: Elaboración de los autores a partir de datos del levantamiento y base cartográfica del DPAE/UFSC

\section{Descripción del espacio y sus relaciones}

En cuanto a las variables independientes, se aplicaron, principalmente, medidas provenientes de la Teoría de la Sintaxis Espacial para describir el espacio y sus relaciones.

Los mapas sintácticos fueron elaborados por los autores, adoptando como unidad espacial las líneas axiales, que son líneas de visión y movimiento usadas para describir el sistema (continuo) de espacios abiertos de un determinado sistema. Su función es proveer una base espacial adecuada para las operaciones de la Sintaxis Espacial relativas al análisis y cuantificación de las relaciones de cada elemento del sistema con todos los otros elementos. Por ese motivo, la Sintaxis se diferencia de otros abordajes más tradicionales, que enfatizan los aspectos locales de cada espacio (Peponis, 1989), como su formato, tamaño y proporción, revelando, en contraste, propiedades de los espacios que solamente su relación con los otros pueden explicar, tales como su mayor o menor profundidad en el sistema (denominada Integración) y su posición en el camino entre otros espacios (denominada Elección).

La nomenclatura utiliza comúnmente la letra " $R$ " para representar el radio de alcance del análisis. Cuando este radio incluye todas las líneas del sistema, se representa con " $n$ "; cuando es usado para representar los efectos en escala local, tradicionalmente es igual a 3 (R3), considerando, así, sólo tres líneas que siguen en cualquier dirección a partir de determinada línea axial (Hillier, 1996).

La integración también se trabajó basada en mapas de permeabilidad. Estos son construidos a través de cuadrículas regulares, en el estilo de las capas raster de 
los Sistemas de Información Geográfica. Cada celda de la cuadrícula es considerada como una unidad espacial, y las celdas mutuamente accesibles, es decir, aquellas que pueden ser directamente recorridas por los transeúntes a través de una línea recta, son consideradas directamente conectadas. A partir de allí, la profundidad topológica de cada celda se calcula exactamente como en la Integración por líneas axiales (Turner et al., 2001).

Para la visibilidad se utilizó el concepto de "isovistas" que, según Benedikt (1979), es el polígono que demarca el área visible a partir de un punto en el espacio. El área total de la isovista, por consiguiente, da una idea de cuánto puede verse desde un punto determinado, y desde cuántos otros puntos este espacio puede verse. Así como el mapa de permeabilidad, el mapa de visibilidad también se elaboró usando una cuadrícula regular de celdas, porque este tipo de representación permite revelar mayores detalles en escalas ampliadas que en las líneas axiales. En él, los tonos más cercanos al rojo indican puntos cuya isovista es mayor, mientras que los puntos más azulados indican isovistas menores (Turner et al., 2001). Adicionalmente, se hicieron algunos mapas con isovistas específicas, elaborados para estudiar el área visible a partir de puntos de interés (Figura 3c, 3d)

La Figura 2 ilustra la ficha de contextualización de uno de los objetivos, con los mapas de Integración y Elección Rn y R3, visibilidad y permeabilidad, y la Figura 3 muestra los mapas de comportamiento, de densidad de apropiación y los estudios de isovistas.

\section{Investigación de reconocimiento de la diversidad}

Para verificar la diversidad de perfiles de usuarios, en cada lugar fueron aplicados pequeños cuestionarios, en los mismos horarios de aquellos utilizados en la investigación del comportamiento. Las preguntas se elaboraron para ser sencillas y de rápida respuesta, respetando el anonimato del entrevistado, y se basaron en el trabajo de Yaylali-Yildiz et al., (2013), que utilizó esta herramienta en el análisis socioespacial de la construcción del campus universitario de Aegean, en Turquía. Las tres preguntas fueron: ¿A qué curso pertenece? ¿Cuál es el lugar que utiliza con más frecuencia para sus actividades extracurriculares o en los intervalos? ¿Cuáles son los motivos para frecuentar este lugar (selección múltiple)? Los motivos podían ser: posibilidad de interacción social; para encontrar amigos de mi curso/sector; para encontrar amigos de otros cursos/sectores; para ver personas/movimiento; porque la calidad/precio del café/comida es mejor; proximidad con el aula/trabajo; porque hay infraestructura para estudiar; comodidad y características físicas (bancos, mesas, apoyos); u otros.

Por medio de un formulario electrónico, tres investigadores aplicaron los cuestionarios en todos los tramos, en 6 días diferentes (entre el 13/04/2017 y el 27/04/2017), totalizando 480 respuestas (60 en cada tramo). Después de recolectados todos los datos, los resultados sobre el curso/centro de pertenencia y los motivos para estar en aquel lugar fueron tabulados y comparados entre tramos a través de proporciones simples $y$, para la diversidad, del índice de Gini-Simpson True Diversity (Ecuación 1). La clasificación de la variable se dividió entre diversidad de cursos y entre centros (unidades administrativas que agrupan cursos y departamentos en áreas específicas del campus universitario), ambas consideradas importantes para la comprensión de la diversidad.

$$
\begin{array}{ll}
\text { a) } D_{G s}=1-\sum_{i=1}^{s} p_{i}^{2} & \text { b) } D_{T D}=\frac{1}{\left(2-D_{G s}\right)}
\end{array}
$$

Ecuación 1 a) Fórmula del índice de Gini-Simpson; b) Fórmula de la True Diversity. Fuente: Jost (2006)

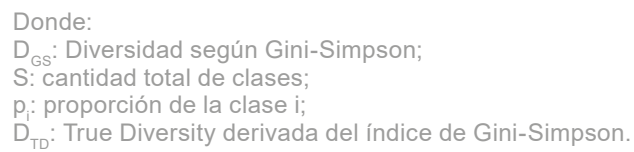

El índice de Gini-Simpson True Diversity equivale a la cantidad de clases diferentes presentes en el conjunto de datos analizados, en caso de que todas ellas tuvieran igual proporción (Jost, 2006). Una muestra con proporciones iguales de usuarios de 5 cursos diferentes, por ejemplo, daría como resultado un índice igual a 5 . En la medida en que las proporciones se vuelven más desiguales, este valor disminuye, como una penalización por desigualdad. En comparación con otros índices más tradicionales de diversidad, tales como el de Shannon y el índice "puro" de Simpson (o Gini-Simpson), la ventaja del Gini-Simpson True Diversity es que su resultado es lineal, lo que quiere decir que un área con índice igual a 4 tiene dos veces más diversidad que un área con índice igual a 2 (Jost, 2006). Eso no ocurre con los índices tradicionales porque ellos incorporan operadores no lineales (logaritmo, en el índice Shannon, y cuadrático, en el caso de Gini-Simpson).

\section{Análisis visual y cuantitativo}

Los datos se analizaron de dos maneras: a) de forma visual y b) a través de correlaciones dentro de los lugares y entre ellos. El análisis visual comparó los mapas producidos en los pasos anteriores, buscando patrones de comportamiento reveladores y posibles relaciones de asociación entre las variables. El examen fue más cualitativo en esa etapa y consideró cada lugar de manera más profunda, con visitas y fotos complementarias para ayudar en la obtención de conclusiones.

Para los análisis de correlación dentro de cada lugar, los valores de las variables dependientes y de las medidas sintácticas se agregaron por tramos, esto es, por porciones de los lugares comprendidos entre dos intersecciones del paso de transeúntes. Para la cuantificación de personas en movimiento o estáticas, se contabilizaron sólo aquellas que se localizaban en el camino representado por la línea axial, o muy próximas a él. Las personas en lugares con pertenencia dudosa a la línea no se consideraron para las correlaciones. Para los valores de visibilidad (área de la isovista) y permeabilidad (integración) se tomó en consideración el eje de la línea axial y 4 unidades de la cuadrícula de celdas a lo largo de la línea, generando un promedio entre sus valores. La Tabla 1 describe las variables utilizadas en esta etapa. 


\begin{tabular}{|c|c|c|}
\hline Variables & Operación & Fuente \\
\hline \multicolumn{3}{|l|}{ Dependientes } \\
\hline Copresencia & Densidad de la apropiación (mapa kernel) & \multirow{4}{*}{ Levantamiento in situ } \\
\hline Personas en movimiento & Mapa de personas en movimiento & \\
\hline Personas estáticas & Mapa de personas paradas & \\
\hline Interacción & Total de personas en parejas o grupos & \\
\hline Diversidad & $\begin{array}{l}\text { Diversidad entre centros (índice de Gini-Simpson True Diversity) } \\
\text { Diversidad entre cursos (índice de Gini-Simpson True Diversity) }\end{array}$ & \multirow[t]{2}{*}{ Cuestionario } \\
\hline Motivo para frecuentar el lugar & Proporción de respuestas para cada motivo & \\
\hline \multicolumn{3}{|l|}{ Independientes } \\
\hline Integración & $\begin{array}{l}\text { Integración global + líneas axiales } \\
\text { Integración lugar (R3) + líneas axiales }\end{array}$ & \multirow{4}{*}{$\begin{array}{l}\text { Base cartográfica + } \\
\text { Depthmap }\end{array}$} \\
\hline Permeabilidad & Integración global + celdas raster $(1,8 \mathrm{~m}$ x 1,8m) & \\
\hline Amplitud visual & Área de las isovistas (mapa de visibilidad) & \\
\hline Visuales & Isovista a partir del área más utilizada & \\
\hline Infraestructura para permanencia & Cantidad de mobiliario (bancos, mesas) & \multirow{2}{*}{ Levantamiento in situ } \\
\hline Puntos de interés & Proximidad con puntos de interés & \\
\hline
\end{tabular}

Tabla 1 Operación y fuente de las variables utilizadas en el análisis dentro de cada lugar. Fuente: Elaboración de los autores, 2017

\begin{tabular}{|c|c|c|}
\hline Variables & Operación & Fuente \\
\hline \multicolumn{3}{|l|}{ Dependientes } \\
\hline Copresencia & Total de personas & \multirow{2}{*}{ Levantamiento in situ } \\
\hline Interacción & Total de personas en parejas o grupos & \\
\hline Diversidad & $\begin{array}{l}\text { Diversidad entre Centros (índice de Gini-Simpson True Diversity) } \\
\text { Diversidad entre cursos (índice de Gini-Simpson True Diversity) }\end{array}$ & Cuestionario \\
\hline \multicolumn{3}{|l|}{ Independientes } \\
\hline \multirow[t]{2}{*}{ Proximidad a otros lugares } & Valor promedio de la Integración global por líneas axiales & \multirow{6}{*}{$\begin{array}{c}\text { Base cartográfica + } \\
\text { Depthmap }\end{array}$} \\
\hline & Valor promedio da Integración lugar (R3) por líneas axiales & \\
\hline \multirow[t]{2}{*}{ Permanecer en el camino } & Valor promedio de la Elección global por líneas axiales & \\
\hline & Valor promedio de la Elección local (R3) por líneas axiales & \\
\hline Amplitud visual & Promedio de las áreas de las isovistas por celdas raster & \\
\hline Permeabilidad & Promedio de las áreas permeables por celdas raster & \\
\hline Mobiliario & Cantidad de Mobiliario (bancos, mesas, apoyos) & Levantamiento in situ \\
\hline
\end{tabular}

Tabla 2 Operación y fuente de las variables utilizadas en el análisis entre lugares. Fuente: Elaboración de los autores, 2017

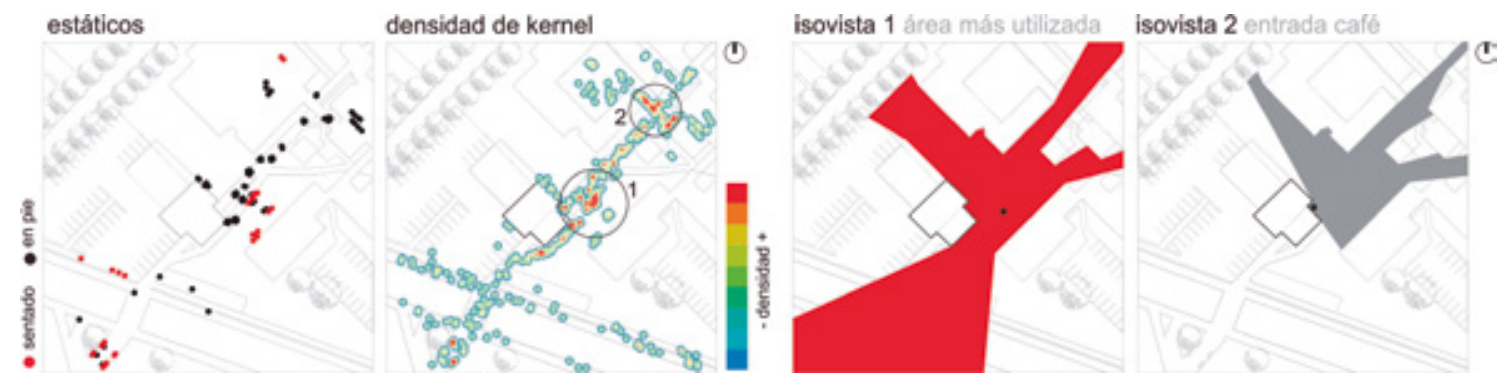

Figura 4 Comportamiento del área 5. De la izquierda para derecha (a): Mapa de personas estáticas. (b)Mapa de densidad de Kernel. (c): Isovista del punto de mayor utilización por los usuarios. (d) isovista de la entrada del café. Fuente: Elaboración de los autores a partir de datos del levantamiento y base cartográfica del DPAE/UFSC 
Al final, se realizó la comparación entre los resultados agregados por lugares, comparándolos entre sí. Para ello, en cada objetivo las variables dependientes e independientes se sintetizaron en un índice para el área como un todo, utilizando la cantidad total, la proporción o el promedio, según el caso (Tabla 2). Para las correlaciones generales entre tramos, se consideró un promedio de todos los valores atribuidos a cada línea axial dentro del área. Lo mismo fue efectuado con los análisis de permeabilidad y visibilidad, considerando un promedio de todas las celdas de la cuadrícula dentro del tramo. En cuanto al mobiliario, se contabilizó todos los bancos, con o sin respaldo, y las mesas presentes en el espacio externo. Para bancos lineales, el valor en metro lineal se divide entre la dimensión de un banco común en el campus, de 1,10 metro.

\section{Limitaciones}

Cabe señalar ahora algunas limitaciones del presente estudio. En primer lugar, se trata de un trabajo sobre un único campus, por lo tanto, la posibilidad de generalización de los resultados debe ser vista con cautela. Entiéndase que otros estudios semejantes deben llevarse a cabo para corroborar o cuestionar los resultados obtenidos. El uso de ocho áreas, por otro lado, permitió verificar recurrencias en las relaciones entre las variables. Sin embargo, no permite una alta confiabilidad en las correlaciones estadísticas, que, por ende, son ofrecidas sólo como indicadores preliminares.

\section{RESULTADOS}

\section{Análisis de los lugares}

La visibilidad analizada en los objetivos, considerada en términos de área total visible en cada punto, fue apenas capaz de prever los lugares exactos en los que hubo mayor cantidad de personas estáticas. Mientras, el formato de la isovista, y las porciones del espacio que esta abarca, posibilitó detectar un patrón claro: gran parte de los usuarios se posicionaban de modo de conseguir visualizar los principales caminos del objetivo, por donde, consecuentemente, pasaba más gente, transformando incluso el espacio para que eso ocurriera. Un ejemplo de aquello lo otorga la Figura 4, en la cual se aprecia que la isovista con la mayor concentración de personas proporciona una vista de casi todos los usuarios al objetivo.

Los sitios que tiene mayor intensidad puntual de personas (así como los señalados con 1 y 2, en la Figura 4b), tienen relación con dos aspectos espaciales: proximidad con uno o más puntos de interés y disponibilidad de infraestructura para la permanencia (mobiliario). Así, los lugares que tenían los dos aspectos en combinación (lugares 1, 2 y 4) tuvieron mayor porcentaje de personas estáticas ( $30 \%$ y más que el resto) y tuvieron al menos un $40 \%$ de sus usuarios interactuando. Allí se encontraban puntos de interés como cafés, copiadoras, servicios académicos, terminales bancarios, sanitarios y accesos a los salones de clase, interconectados por pasillos o áreas externas abiertas y cubiertas, donde existen espacios que son objeto de apropiación y flexibilización (pequeños eventos, exposiciones, o reunión de grupos). La disponibilidad de mobiliario, aliada a una configuración delimitada con potencial visual hacia las áreas de gran movimiento, fue otro rasgo detectado en todos los objetivos, donde aumenta la cantidad de personas estáticas, en grupos o en parejas, que conforman pequeños grupos y círculos de conversación (Figura 5). Ninguno de estos tres lugares posee patrones claros en relación a los análisis sintácticos: mientras el área 2 está bien integrada global y localmente, las áreas 1 y 4 no obtuvieron valores tan altos, a causa de su localización limítrofe del campus.
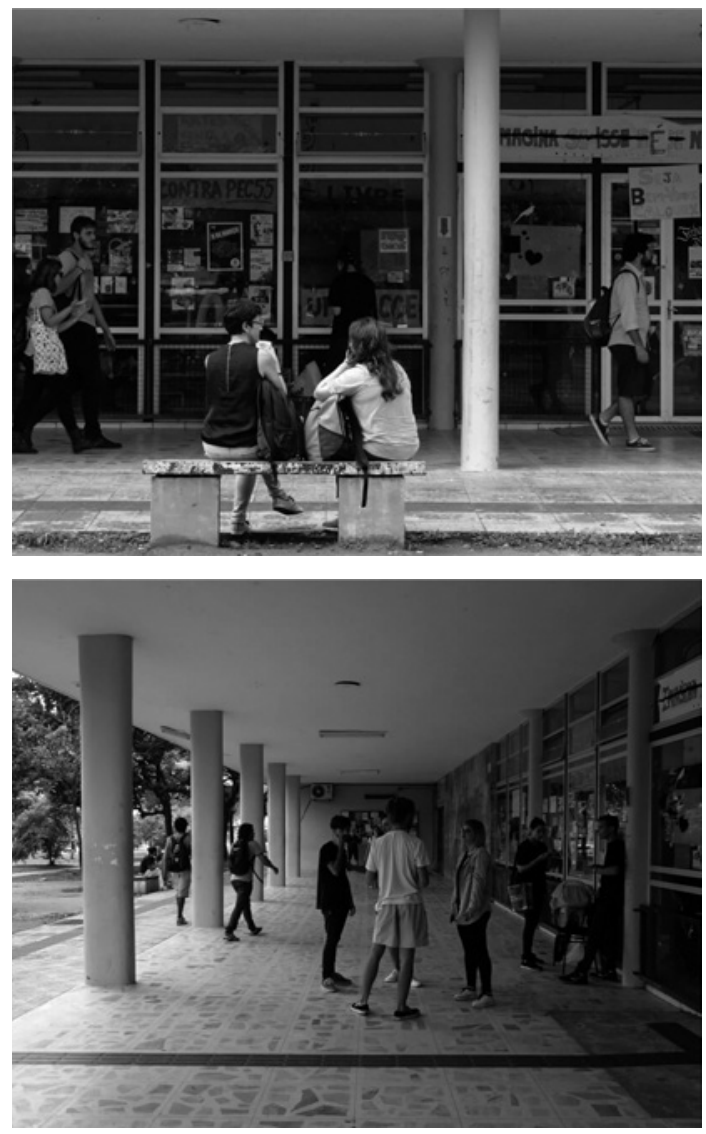

Figura 5 Fotos del tramo 2, apropiación de los estudiantes. Fuente: Fotografías de los autores, 2017 


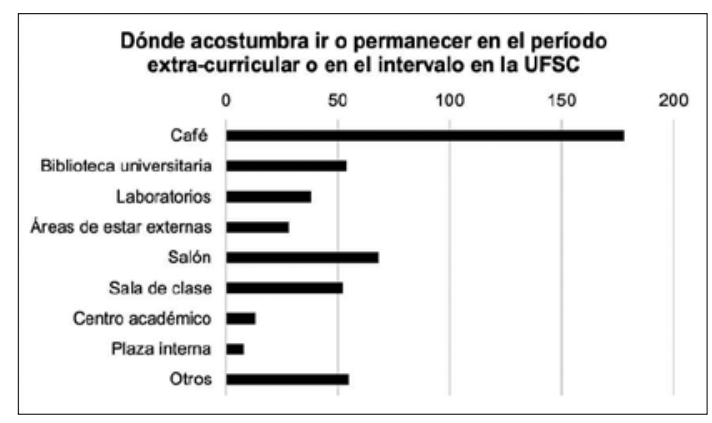

Figura 6 Resultados generales de los cuestionarios para la pregunta: Lugar al que acostumbra ir o permanecer en el período extracurricular en la Universidad Universidad Federal de Santa Catarina, Brasil. Fuente: Elaboración de los autores en base a datos del cuestionario, 2017.

Tal como afirman las investigaciones de Penn, Desyllas y Vaughan (1999), Allen y Henn (2013), y Kabo et al. (2015), el área de los cafés se presenta como un espacio importante para intercambiar y compartir la información. Efectivamente, en los lugares analizados aquí y en las áreas próximas a las entradas de los cafés se constató una interacción más intensa y con mayor número de personas estáticas de pie que en otras áreas. Conectar estos puntos de interacción a los caminos de gran flujo y otros servicios se vuelve, entonces, esencial para fomentar las interacciones. Asimismo, los espacios que ofrecen pocas oportunidades tienden a impedir la permanencia de las personas, pues no hay elementos suficientes que la sustenten.

Estos resultados también aparecen evidenciados en los gráficos de lugares de preferencia y motivaciones realizados junto con la investigación de reconocimiento de diversidad. Los resultados de los cuestionarios demostraron que el principal lugar de permanencia extracurricular corresponde a los cafés, seguido de los pasillos (Figura 6), los cuales motivan a los alumnos a salir de los espacios formales de aprendizaje y auspician los encuentros informales y la copresencia. El principal motivo de elección, según las entrevistas, es la proximidad del lugar, seguido de la posibilidad de interacción social y la oportunidad de encontrar amigos de su mismo curso (Figura 7). Es pertinente notar que la opción de encontrar amigos de otros cursos no tuvo resultado significativo, ya que posiblemente las personas no se mueven tan lejos para buscar personas de otras áreas, prefiriendo las áreas cercanas. Es allí donde entra el papel de la configuración espacial de aproximar personas de otros cursos y disciplinas a través de un campus multidisciplinario que favorezca el surgimiento de vínculos débiles a través de la distribución y mezcla de movimientos. Otro resultado interesante es que los lugares donde la opción "aulas de clase" obtuvo alto número de respuestas ( 5 y 8 ) fueron aquellos en los que el café no estaba integrado al flujo del movimiento existente o no tenía un área física fija, lo cual refuerza la necesidad detectada de que estos elementos funcionen en conjunto para fomentar el encuentro y la interacción.

El resultado de la proximidad como un factor importante para los usuarios en la elección del lugar extracurricular explica los bajos valores de diversidad en los perfiles de usuarios, entre centros y cursos (Tabla 3), que prefieren permanecer en su departamento. Aunque sea bajo, entre los centros, los mayores valores se dan en los lugares 2 y 8 : el primero, que comprende el área más céntrica del campus, frente a la Plaza de la Ciudadanía, y el otro que se localiza en un acceso importante para la universidad. En cuanto a la diversidad entre cursos, las áreas que tuvieron mayor cantidad de diversidad ( 6 y 7) están bien conectadas en ambas medidas sintácticas, de Integración ( $R n$ y R3) y Elección (Rn), y tienen espacios bien delimitados por plazas que distribuyen áreas de cafés y servicios, conformando corredores sociales importantes que conectan con el centro del campus, donde se reúnen estudiantes de diferentes cursos, pertenecientes al mismo centro. Para la diversidad, las áreas de tránsito y trayectos hacia los principales edificios y servicios académicos del campus, como la Rectoría, la Biblioteca y el Centro de Eventos, se revelan importantes en la construcción de áreas interdisciplinarias.

Los resultados de los análisis espaciales sintácticos, en correlación con las variables de comportamiento, demostraron que las personas en movimiento y estáticas, están mejor representadas por los atributos locales (R3), tanto de elección como de integración (Figura 8 y 9). De manera general, los análisis de movimiento tienen mejores resultados, ya que poseen relación directa con la manera en que los caminos se configuran y conectan, facilitando que los análisis sintácticos capturen estos flujos de movimiento. En el caso de las personas estáticas, a pesar de que encontremos resultados satisfactorios para las medidas sintácticas, elementos como proximidad a los servicios, una definición adecuada del espacio, visibilidad y mobiliarios, por ejemplo, (o sea, aspectos locales del espacio) parecen ser más relevantes para los lugares en que las personas se detienen durante sus jornadas, como se vio anteriormente.

Los tres lugares con mejor correlación, tanto para el movimiento como para las personas estáticas (1, 6 y 7), son áreas con altos valores de integración en un todo del campus, tienen caminos bien definidos por medio de plazas y son accesos importantes para sus departamentos, con oferta de servicios a lo largo del camino (Figura 10). Por el contrario, los lugares 5 y 8 , que presentan las peores correlaciones, están poco integrados globalmente y se sitúan en los límites del campus, teniendo como destino solo su propio departamento. Es posible que los análisis sintácticos no consigan captar la dinámica de esos tramos por dos motivos: porque presentan flujo de movimiento y personas estáticas en sus caminos resultantes de las actividades de su departamento, aunque sus valores sintácticos globales sean bajos; y porque están compuestos por pocas líneas axiales (8;12 líneas), concentrando todo el flujo en sólo una o dos de ellas y explicando también las bajas correlaciones con medidas locales. 


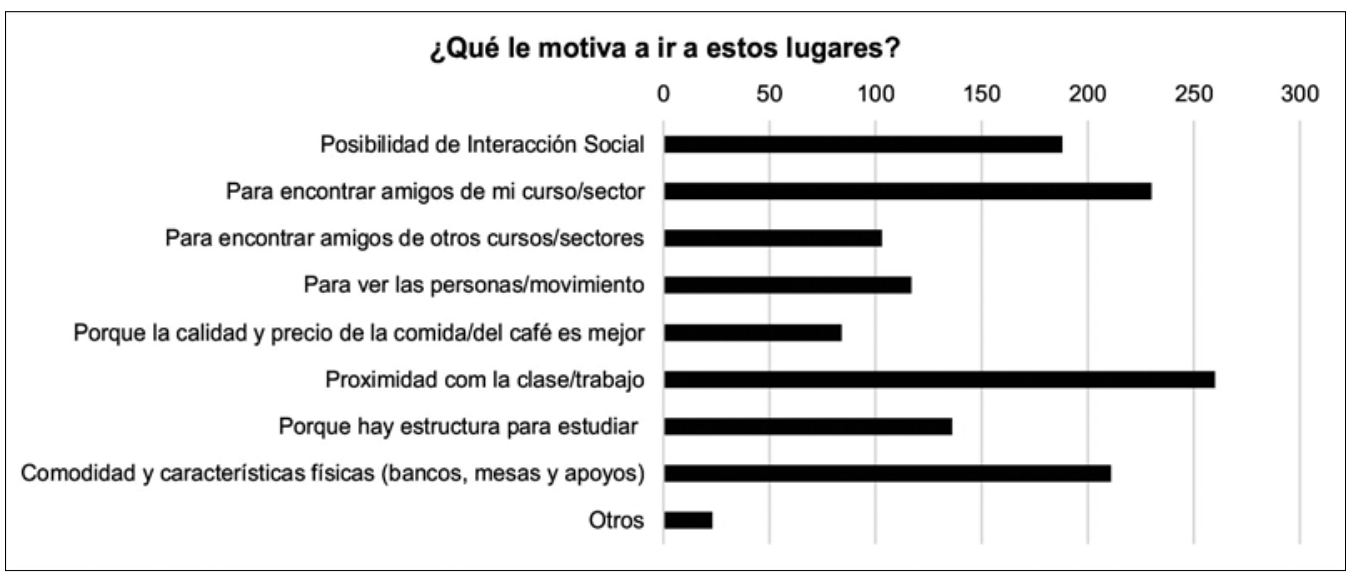

Figura 7 Resultados generales de los cuestionarios para la pregunta: ¿Qué le motiva a ir a estos lugares? Fuente: Elaboración de los autores, 2017

\begin{tabular}{c|cccccccc} 
Área & $\mathbf{1}$ & $\mathbf{2}$ & $\mathbf{3}$ & $\mathbf{4}$ & $\mathbf{5}$ & $\mathbf{6}$ & $\mathbf{7}$ & $\mathbf{8}$ \\
\hline GinitD Centros (1-15) & 2,817 & $\mathbf{3 , 1 6 6}$ & 2,468 & 1,375 & 1,807 & 1,478 & 1,434 & $\mathbf{5 , 3 0 4}$ \\
GiniTD Cursos (1-52) & 6,951 & 7,724 & 8,999 & 7,258 & 6,292 & $\mathbf{1 0 , 1 7 2}$ & $\mathbf{1 0 , 3 0 5}$ & 5,845
\end{tabular}

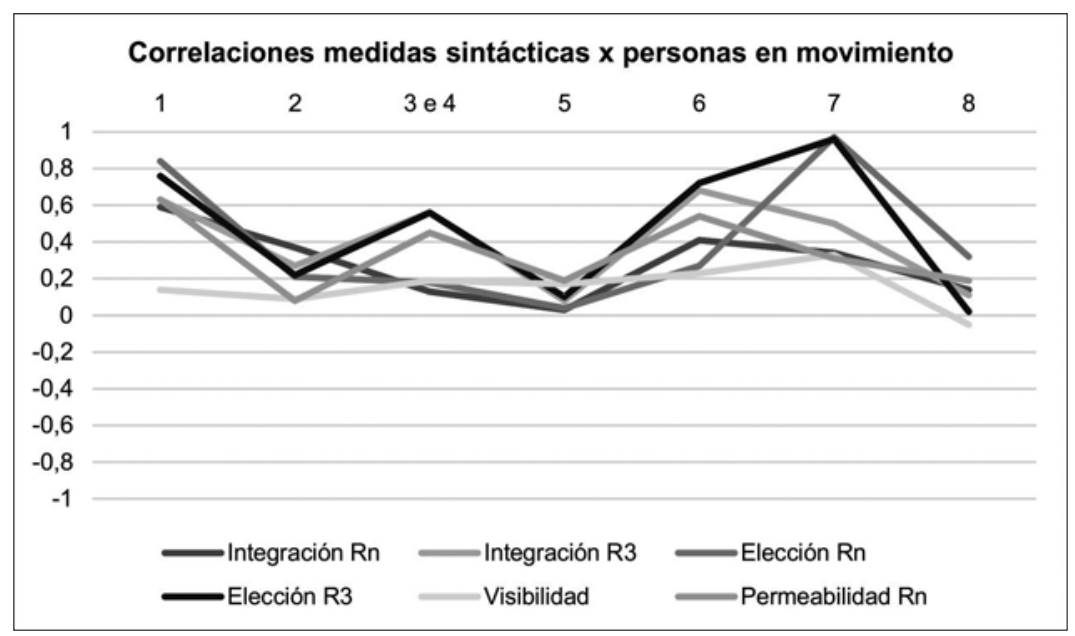

Figura 8 Correlaciones entre las medidas sintácticas y las personas en movimiento. Fuente: Elaboración de los autores, 2017 


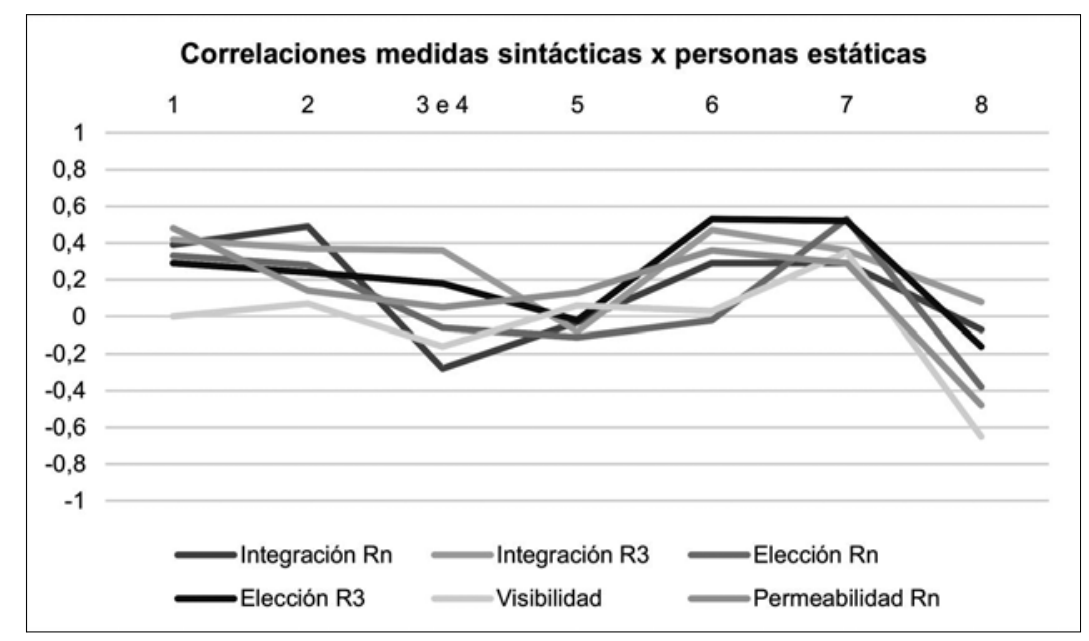

Figura 9 Correlaciones entre las medidas sintácticas y las personas estáticas. Fuente; Elaboración de los autores, 2017
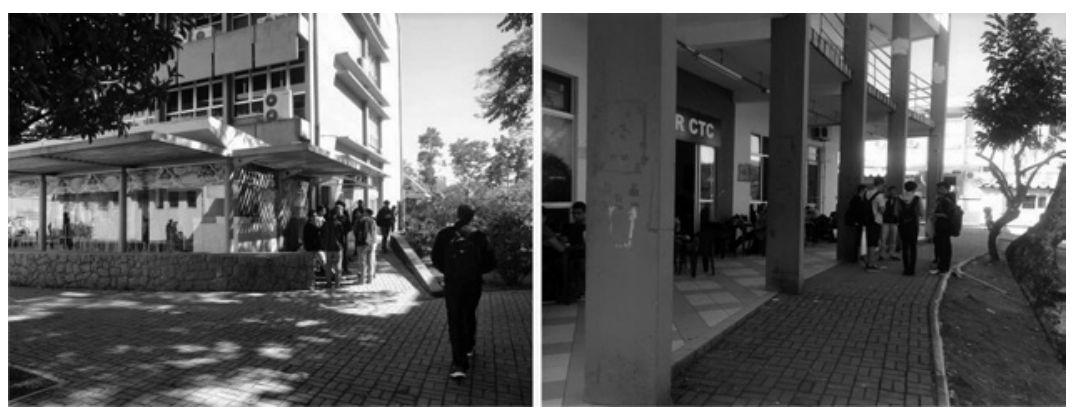

Figura 10 A la izquierda: Área 6, en la cual se observan alumnos interactuando cerca de la entrada del café. A la derecha: Área 7, en la cual se observa reunión de grupos frente al café. Fuente: Fotografías de los autores, 2017

\section{Cruce entre las variables y los ocho objetivos}

En el cruce de datos para los ocho lugares (Tabla 4), las variables de copresencia e interacción tuvieron correlaciones semejantes cuando se relacionaron con las variables independientes. La primera confirmación de esta investigación apuntó que, cuanto mayor es la copresencia, tanto mayor será la interacción. Esto, a pesar de parecer obvio, es un resultado significativo, pues refuerza la noción de que la copresencia es una base importante $-y$ efectiva- para interacciones más profundas. Dicho esto, las medidas que mejor captaron ambas variables fueron las de Integración y Elección local (con hasta 3 pasos topológicos) y, con menos intensidad, la medida global de Integración (Rn). Ello sugiere que, cuanto más integrado localmente, mayores son las posibilidades de movimiento de personas $y$, principalmente, de interacción, dada la correlación respectiva de 0,817 ; con la salvedad de que todas las áreas estudiadas en este trabajo incluyen un punto de interés relativamente importante en su centro. Con todo, es posible hacer otra afirmación: cuanto más segregada es el área globalmente, mayores son las medidas locales que consiguen captar el movimiento y la interacción. Eso vale especialmente para la permeabilidad, que difícilmente tiene resultados positivos en análisis de mayor escala.

En general, los análisis de permeabilidad y visibilidad no se mostraron tan eficaces, y tuvieron sus mayores correlaciones con el movimiento de las áreas más segregadas globalmente.

Por otra parte, el resultado de los análisis de diversidad mostró que la configuración a través de bloques aislados, a la que responde este campus, no favorece los intercambios interdisciplinarios 


\begin{tabular}{|c|c|c|c|c|c|}
\hline \multicolumn{6}{|c|}{ Variables dependientes } \\
\hline \multirow{8}{*}{ 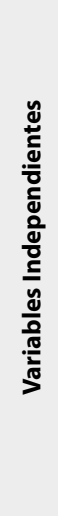 } & & 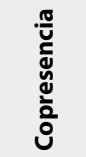 & 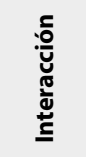 & 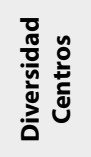 & 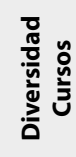 \\
\hline & Integración Rn & 0,517 & 0,517 & $-0,359$ & 0,723 \\
\hline & Integración R3 & 0,651 & 0,817 & $-0,604$ & 0,573 \\
\hline & Elección Rn & $-0,293$ & $-0,402$ & 0,735 & $-0,096$ \\
\hline & Elección R3 & 0,544 & 0,765 & $-0,469$ & 0,794 \\
\hline & Permeabilidad & 0,259 & 0,103 & 0,301 & 0,057 \\
\hline & Visibilidad & $-0,284$ & $-0,607$ & 0,871 & $-0,452$ \\
\hline & Mobiliario & 0,725 & 0,775 & $-0,504$ & 0,281 \\
\hline
\end{tabular}

Tabla 4 Correlaciones entre las variables dependientes y las independientes para los ocho lugares. Valores en rojo indican las mejores correlaciones. Fuente: Elaboración de los autores, 2017
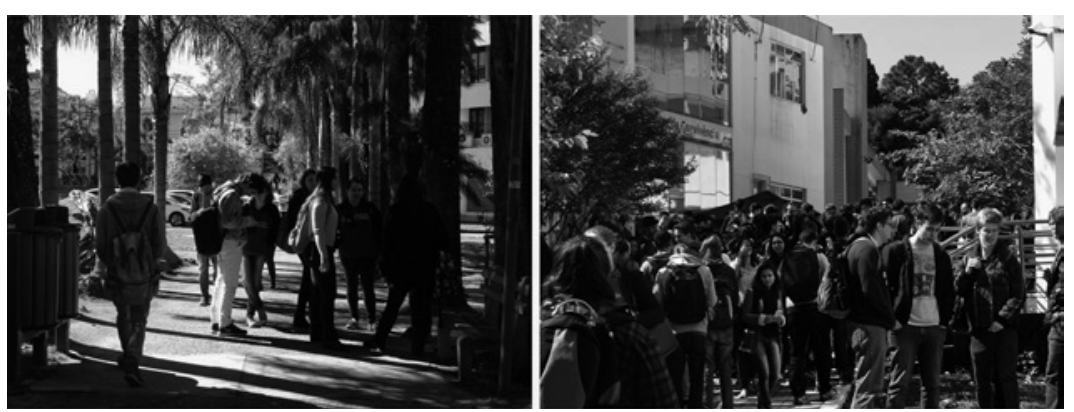

Figura 11 Fotos del área 4, uno de los accesos al área central del campus, con valores altos de Integración y Elección. Fuente: Elaborado por los autores, 2017

entre centros, posiblemente debido a los grandes desplazamientos que serían necesarios entre ellos, teniendo una correlación positiva con la medida de Visibilidad y Elección global (Rn). Para la visibilidad, los resultados alcanzados son singulares y restringidos a características muy específicas de este campus. Los dos objetivos que destacan de los demás en esa correlación (8 y 2 - Tabla 3), tienen áreas de visibilidad grandes, la primera en consecuencia de las áreas deportivas, en su mayoría gramados, y la segunda debido a la plaza de la ciudadanía. Es improbable, sin embargo, para el objetivo 8 , que la visibilidad sea la razón de la diversidad de usuarios, siendo más probable ser fruto de la localización del tramo que sirve como acceso al campus. Para comprobar, cuándo retiramos esta área del análisis la correlación cae de 0,87 a 0,42, que, aunque sea un valor significativo, demuestra la poca robustez de los resultados. En el caso del lugar 2 tiene características comunes en los campus universitarios con las configuraciones que tienen plazas centrales, por lo tanto, es más sustentable su correlación.

Para la diversidad entre cursos, los resultados con mayores correspondencias fueron con las medidas locales de Elección (R3), seguidas de las de Integración (RN e R3). Posiblemente, por cuenta de que los desplazamientos entre cursos están más próximos y ocurren con más frecuencia, las medidas sintácticas consiguen captar mejor el flujo de movimiento entre estas áreas. Por lo tanto, áreas con valores más altos de Integración y Elección (R3), poseen más probabilidad de tener diversidad, en relación a los perfiles de sus usuarios, estimulando las interacciones interdisciplinarias dentro de un mismo centro (Figura 11). 
Reconocer la importancia de calificar y organizar espacios de manera que fortalezcan los vínculos débiles demostró ser esencial en la planificación de las universidades que buscan estudiantes innovadores y creativos. Se percibe que cuando están cercanos las áreas de movimiento y los puntos de interés (en especial con la influencia de áreas de cafés) crean un significativo e influyente centro de gravitación que soporta intensamente la copresencia y la interacción con patrones de comportamiento parecidos.

A pesar de que los análisis de permeabilidad y visibilidad no presenten resultados tan significativos, es perceptible la preferencia de los usuarios de utilizar áreas con alcances visuales estratégicos, principalmente direccionados a las áreas más integradas y con mayor flujo de transeúntes. Además de eso, este artículo demuestra que las áreas más integradas local y globalmente tienen mayores probabilidades de tener alta intensidad de personas, interactuando y con mayor diversidad de usuarios (entre cursos), a pesar de que la medida local represente mejor la dinámica de los tramos.

Conforme hemos visto en la investigación, pocos fueron los entrevistados que pertenecían a otros Centros, mientras que, en relación a los cursos, el resultado demostró mayor diversidad. Para el primero, la Elección Global fue la que mostró mejor correlación, mientras la medida de Elección local fue la que mejor captó la diversidad de cursos dentro de cada lugar analizado. Así, se nota que el mayor nivel de desplazamiento es entre áreas cercanas, evidenciando el factor de Proximidad que parece ser determinante para la coexistencia y consciencia de estímulo de las interacciones interdisciplinares y, en consecuencia, la importancia de pensar en espacios que promuevan la integración y conexión espacial en la escala local. Más específicamente, se reveló como importante una coherente distribución de las actividades que estimulan la permanencia y la interacción, la infraestructura de apoyo para que esto ocurra, y, especialmente, la localización de estos elementos en relación a "permanecer en el camino" entre localizaciones dentro de un radio limitado. Los caminos principales sin estos elementos se vuelven solo un pasillo; elementos como estos en caminos sin importancia dejan de alcanzar su potencial de interacción y encuentro.

De esta forma, los espacios externos necesitan ser fortalecidos para que sean apropiados por la mayor cantidad de personas posible $y$, para ello, deben conectarse mediante corredores sociales y atractivos que funcionen como apoyo para la interacción. Se sugieren estructuras universitarias con menores desplazamientos entre los distintos departamentos, lo que puede ser logrado no sólo por proximidad lineal, euclidiana, sino por caminos que minimicen los cambios de dirección y posibiliten la visibilidad entre los ambientes. Esto disminuye no sólo la distancia física sino también el esfuerzo cognitivo necesario para ir de un lugar a otro, o incluso sólo para tomar consciencia de que un determinado lugar existe y es accesible. El campus requiere un espacio calificado para reproducir el conocimiento, liberándose de los métodos tradicionales de enseñanza y compartiendo información a través del debate interdisciplinario. La educación siempre será un acto espacial.

Reconocimientos: Esta investigación tuvo el apoyo de la Beca Capes.
ABU-GHAZZEH, Tawfiq M. Communicating behavioral research to campus design: Factors affecting the perception and use of outdoor spaces at the University of Jordan. Environment and Behavior, 1999, vol. 31, n 6, pp. 764-804.

ADHYA, Anirban. Evaluating the campus-downtown relationship. The Spatial configuration of four college towns in small metropolitan regions in the United States. Proceedings of the Space Sintax 7th International Symposium, 2009, n 32, pp. 1-19.

ALEXANDER, Cristopher; ISHIKAWA, Sara y SILVERSTEIN, Murray. A pattern language. New York: Oxford University Press, 1977.

ALLEN, Thomas J. Managing the flow of technology. Cambridge, MA: MIT Press, 1977.

ALLEN, Thomas J. y HENN, Gunter W. The Organization and Architecture of Innovation - Managing the Flow of Technology. Oxford, UK: Elsevier, 2013.

BENEDIKT, Michael. To take hold of space: Isovists and isovists fields. Environment and Planning B, 1979, vol. 6, pp. $47-65$.

BOARNET, Marlon G.; FORSYTH, Ann; DAY, Kristen J. y OAKES, Michael. The Street Level Built Environment and Physical Activity and Walking Results of a Predictive Validity Study for the Irvine Minnesota Inventory. Environment and Behavior, 2011, vol. 43, n 6, pp. 735-775.

CALVO-SOTELO, Pablo Campos. 10 Principles for an innovative model for the 21 St Century university: The Educacional Campus. Aula, 2010, nº 16, pp. 187-200.

CALVO-SOTELO, Pablo Campos. Innovative Educacional Spaces: Architecture, Art and Nature for University ExceIlence. Aula, 2014, n²0, pp. 159-174.

CERVERO, Robert y DUNCAN, Michael. Which Reduces Vehicle Travel More: Jobs-Housing Balance or RetailHousing Mixing? Journal of the American Planning Association, 2006, vol. $72, n^{\circ} 4$, pp. 475-490.

DPAE - Departamento de projetos de arquitetura e engenharia. O planejamento físico na UFSC [en línea] [Consultado 20 enero 2017]. Disponible en: http:// dpae.seoma.ufsc.br/files/2014/05/planejamentoespa\%C3\%A7o-f\%C3\%ADsico_UFSC_Trindade_1960a-2012.pdf

EWING, Reid y CERVERO, Robert. Travel and the Built Environment. Journal of the American Planning Association, 2010, vol. 76, n³, pp. 265-294.

GEHL, Jan. Life between buildings. Washington: Island Press, 2011.

GOFFMAN, Erving. Behavior in Public Places. New York: The Free Press, 1963.

GRANOVETTER, Mark. The Strength of Weak Ties. American Journal of Sociology, 1973, vol. 78, $\mathrm{n}^{\circ}$ 6, pp. 1360-1380. 
GREENE, Margarita y PENN, Alan. Socio-spatial analysis of four university campuses: the implications of spatial configuration on creation and transmission of knowledge. Space Sintax First International Symposium, 1997, vol. 1, n 13, pp. 1-15.

HALL, Edward T. The hidden dimension. Garden city, NY: Doubleday, 1966.

HALSBAND, Frances. Campuses in Place. Places, 2005, n 17, pp. 4-11.

HERTZBERGER, Herman. The Schools of Herman Hertzberger. 10a ed. Rotterdam: Publishers, 2009.

HILLIER, Bill. Space is the machine: A configurational theory of architecture. Cambridge: Cambridge University Press, 1996.

HILLIER, Bill y HANSON, Julienne. The social logic of space. Cambridge: Cambridge University Press, 1984.

HILLIER, Bill; BURDETT, Richard, PEPONIS, John y PENN, Alan. Creating life: or, does Architecture determine anything? Architecture et Comportement/Architecture and Behaviour, vol. 3, n³, p. 233-250, 1987.

HILLIER, Bill; PENN, Alan, HANSON, Julienne, GRAJEWSKI T. y XU, Jian-Ping. Natural movement: or, configuration and attraction in urban pedestrian movement. Environment and Planning B, 1993, vol. 20, pp. 29-66.

HILLIER, Bill y IIDA, Shinichi. Network and psychological effects in urban movement. International conference on spatial information theory, 2005, vol. 3693, pp.475-490.

JACOBS, Jane. The Death and life of great american cities. $4^{\mathrm{a}}$ ed. São Paulo: Martins Fontes, 2009. $1^{\text {a }}$ ed. 1961.

JOST, Lou. Entropy and diversity. Oikos, 2006, no 113, pp. 363-375.

KABO, Felichism; HWANG, Yongha; LEVENSTEIN, Margaret; OWEN-SMITH, Jason. Shared paths do the lab: A sociospatial network analysis of collaboration. Environment and Behavior, 2015, v. 47, pp. 57-84.

LEE, Yumi; HAN, Gwang Ya y KIM, Hong-ill. The UniversityCity Interface : Plazas and Boulevards. Journal of Building Construction and Planning Research, 2014, no 2, pp. 157-165.

PENN, Alan; HILLIER, Bill; BANISTER, David y XU, Jian-Ping. Configurational modelling of urban movement networks. Environment and Planning B: Planning and Design, 1998, vol. 25, n. 1, pp. 59-84.

PENN, Alan; DESYLLAS, Jake y VAUGHAN, Laura. The space of innovation. Environment and Planning B: Planning and Design, 1999, vol. 26, pp. 193-218.

PEPONIS, John. Space, Culture and urban design in the late modernism and after. Ekistics, 1989, n 56, pp. 93-108.

PEPONIS, John; BAFNA, Sonit; BAJAJ, Ritu; BROMBERG, Joyce; CONGDON, Chrsitine; RASHID, Mahbub; WARMELS, Susan; ZHANG, Yan; ZIMRING, Craig. Designing Space to Support Knowledge Work. Environment and Behavior, 2007 , v. 39 , nº, pp. $815-840$
GEHRKE, Steven y CLIFTON, Kelly. Operationalizing Land Use Diversity at Varying Geographic Scales and Its Connection to Mode Choice. Transportation Research Record: Journal of the Transportation Research Board, 2014, vol. 2453, pp. 128-136.

SABOYA, Renato; BITTENCOURT; Sofia; STELZNER, Mariana; SABBAGH, Caio y BINS ELY, Vera. Padrões de visibilidade e permeabilidade em espaços públicos abertos: um estudo sintático em Curitiba - PR. Arquitextos - Vitruvius, 2014, n.164.1.

SMITH, Benjamin y WILSON, J. Bastow. A Consumer's Guide to Evenness Indices. Oikos, 1996, vol. 76, n 1, pp. $70-82$

SONG, Yan; MERLIN, Louis y RODRIGUEZ, Daniel. Comparing measures of urban land use mix. Computers, Environment and Urban Systems, 2013, vol. 42, $n^{\circ}$ Supplement C, pp. 1-13.

SOSA, Manuel E. Where do creative interactions come from? The role of tie content and social networks. OrganizationScience, 2011, vol. 22, n¹, pp. 1-21.

TALEN, Emily. Design that enables diversity: The complications of a planning ideal [en línea]. Journal of Planning Literature, 2006, vol. 20, $n^{\circ} 3,233-249$. DOI: $10.1177 / 0885412205283104$

TALEN, Emily. Design for diversity: Exploring socially mixed neighborhoods. Oxford: Architectural Press, 2008.

TURNER, Alasdair; DOXA, Maria; O'SULLIVAN, David y PENN, Alan. From isovists to visibility graphs: a methodology for the analysis of architectural space. Environment and Planning B: Planning and Design, 2001, vol. 28, pp. 103-121.

VAN DYCK, Delfien; CERIN, Ester; L CONWAY, Terry; BOURDEAUDHUIJ, Ilse; OWEN, Neville; KERR, Jacqueline; CARDON, Greet; FRANK, Lawrence D.; SAELENS, Brian E. y SALLIS, James F. Perceived neighborhood environmental attributes associated with adults' transport-related walking and cycling: Findings from the USA, Australia and Belgium. International Journal of Behavioral Nutrition and Physical Activity, 2012, vol. 9, pp. 70.

WHYTE, William $\mathrm{H}$. The social life of small urban spaces. New York: Project for Public Spaces, 1980.

WINEMAN, Jean; HWANG, Yongha; KABO, Felichism; OWEN-SMITH, Jason y DAVIS, Gerald F. Spatial layout, Social structure, And innovation in organizations. Environment and Planning B: Planning and Design, 2014, vol. $41, n^{\circ} 6$, pp. 1100-1112.

YAYLALI-YILDIZ, Berna; ÇIL, Ela; CAN, Isin y KILIÇ-ÇALGI$\mathrm{Cl}$, Pinar. Analyzing the socio-spatial construction of a university campus: Aegean university as public space of student community. En: Proceedings of the Space Sintax 9th International Symposium, 2013, no 104, pp. 1-18.

ZEISEL, John. Inquiry by Design: Tools for environment behavior research. Cambridge: Cambridge University Press, 1984. 